\title{
THE OCCURRENCE OF REACTIVE OXYGEN SPECIES IN THE SEMEN OF MALES FROM INFERTILE COUPLES
}

\author{
Jiří Novotnýa, Ivana Oborná ${ }^{b *}$ Jana Březinováb, \\ Magda Svobodováb, Jan Hrbáčc , Helena Fingerová ${ }^{b}$
}

\author{
a Institute of Biology, Faculty of Medicine, Hněvotínská 3, Palacký University, 77515 Olomouc, Czech Republic \\ b Clinic of Obstetrics and Gynaecology, Faculty of Medicine, I. P. Pavlova 22, Palacký University, \\ 77515 Olomouc, Czech Republic \\ c Department of Physical Chemistry, Faculty of Science, tr. Svobody 26, 771 46, Palacký University, \\ Olomouc, Czech Republic
}

Received: October 12, 2003; Accepted: October 30, 2003

Keywords: Reactive oxygen species (ROS) / Oxidative stress / Spermatozoa / Leukocytes / Chemiluminescence

The aim of this pilot study was to establish a reactive oxygen species (ROS) evaluation method as a step in the routine diagnosis of men from infertile couples, which attend the Centre of Assisted Reproduction at the Teaching Hospital in Olomouc. Standard semen analyses were performed manually according to WHO guidelines. The number of peroxidase-positive leukocytes in the semen was determined using the Endtz test. The levels of ROS were estimated by chemiluminescence assay using luminol (5-amino-2,3 dihydro-1,4 phthalazinedione) as a probe. The semen samples were collected from 68 patients. Normospermia was found in 15 patients $(22.1 \%)$. The semen samples of 3 normospermic patients were classified as ROS-positive. Elevated ROS production was recorded in all subgroups of patients irrespective of any pathology found. We confirmed that spermatozoa might be the source of ROS as well as the seminal leukocytes. Apart from the leukocytes, sperm cells with residual cytoplasm and immature spermatozoa are considered to be a major source of ROS. Thus it is suggested that sperm morphology abnormalities should be evaluated more carefully.

\section{INTRODUCTION}

Male gametes are highly specialized and differentiated cells designed for a single purpose, for the fertilization of the oocyte. There is a substantial body of evidence suggesting that human semen is getting poorer with the passage of time ${ }^{19}$. Sperm concentration is decreasing and the numbers of defective sperm as well as other male pathologies are increasing. Aitken ${ }^{1}$ reported that up to $80 \%$ of ejaculated spermatozoa are found to be morphologically defective, even in normal fertile males.

Cells living under aerobic condition constantly face the oxygen paradox: oxygen is indispensable for supporting life, but its metabolites such as reactive oxygen species (ROS) can modify cell function. Free radicals may have beneficial or detrimental effects on sperm functions, depending on their nature and concentration ${ }^{2}$. Oxidative stress (OS) plays an important role in human reproduction; OS arises as a consequence of excessive ROS production and/or impaired antioxidant defence mechanisms.

Owing to their deleterious effects on human spermatozoa, excessive ROS must be continuously inactivated to keep only a small amount necessary to maintain normal cell function ${ }^{3,4}$. The plasma membrane of spermatozoa is particularly susceptible to ROS induced damage because of its high content of polyunsaturated fatty acids (PUFA $)^{5,6}$. Sperm cytoplasmic volume is very low and its cytoplasm contains only low concentrations of free radical scavenging enzymes ${ }^{7}$.

In contrast, seminal plasma is well endowed with an array of antioxidant defense mechanism to protect spermatozoa against oxidants and this compensates for the deficiency in cytoplasmic enzymes in the spermatozoa ${ }^{4}$.

However, pathological levels of ROS detected in the semen of infertile men are probably caused by increased ROS production rather than reduced antioxidant capacity of the seminal plasma. The presence of excess residual cytoplasm (or cytoplasmic droplets) is a major determinant of ROS generation by these defective spermatozoa. Oxidative stress-mediated damage to the sperm plasma membrane may account for defective sperm function observed in a high proportion of infertility patients ${ }^{2}$. Oxidative stress-induced DNA damage may accelerate the process of germ cell apoptosis leading to the decline in sperm counts observed in the $20^{\text {th }}$ century ${ }^{4}$.

The aim of this study was to establish ROS evaluation method as a step in the routine examination of men from infertile couples at the Teaching Hospital in Olomouc. 


\section{MATERIALS AND METHODS}

Semen samples were collected from 68 men attending the Centre for Assisted Reproduction of the Teaching Hospital in Olomouc. Only patients from infertile couples were included in this study. All patients were examined only once.

\section{Semen analysis}

Semen samples were collected by masturbation after a period of 3 to 5 days of sexual abstinence. After liquefaction $\left(37^{\circ} \mathrm{C}, 30 \mathrm{~min}\right)$, standard semen analysis was performed manually according to WHO guidelines: semen samples with sperm concentration $\geq 20 \times 10^{6} / \mathrm{ml}$, motility $\geq 50 \%$, morphology $\geq 30 \%$, WBC $\leq 1 \times 10^{6} / \mathrm{ml}$ were considered to be normospermic. Number of peroxidase-positive leukocytes in the semen was determined using the Endtz test ${ }^{9}$.

\section{Endtz test}

A $20 \mu \mathrm{l}$ volume of liquefied semen specimen was mixed together with $20 \mu \mathrm{l}$ of phosphate buffered saline (PBS; pH 7.0) and $40 \mu 1$ of benzidine (4,4'-diaminobiphenyl) solution ${ }^{10}$. The mixed solution was allowed to settle at room temperature for $5 \mathrm{~min}$. Peroxidase-positive leukocytes staining brown were counted.

\section{Reactive oxygen species (ROS) measurement}

Aliquots of liquefied semen were centrifuged at $300 \mathrm{~g}$ for $7 \mathrm{~min}$. The sperm pellet was washed with PBS and resuspended in PBS. The sperm concentration in samples was adjusted to $2 \times 10^{6} / \mathrm{ml}$ (if ejaculate contained sufficient amount of spermatozoa). Concentration of $5 \times 10^{6} / \mathrm{ml}$ of sperm cells was accepted as the lower limit. Levels of ROS were estimated by chemiluminescence assay using luminol (5-amino-2,3-dihydro-1,4-phthalazinedione) as a probe ${ }^{10} .5 \mathrm{mM}$ solution of luminol diluted in dimethyl sulfoxide (DMSO) was added to the washed sperm suspension in a ratio $10 \mu 1: 400 \mu 1$. Measurements were made using luminometer Digene DCR-1. Levels of ROS were determined by measuring chemiluminescence for $15 \mathrm{~min}$ and the results were expressed as relative light units (RLU). If the sperm concentration ranged between 5 and $20 \times 10^{6} / \mathrm{ml}$ a luminescent signal was calculated to the level of sperm concentration of $20 \times 10^{6} / \mathrm{ml}$.

\section{RESULTS}

15 out of 68 samples ( $22.1 \%$ ) were normospermic according to WHO criteria, in 53 semen samples ( $77.9 \%)$ some pathologic findings were revealed (Tab. 1).
Table 1. Patients distribution to subgroups according to WHO criteria for semen analysis.

\begin{tabular}{|l|c|}
\hline Diagnosis & \% of patients \\
\hline Normospermia & 22.1 \\
\hline Oligospermia & 4.4 \\
\hline Astenospermia & 38.2 \\
\hline Oligo-astenospermia & 2.9 \\
\hline Oligo-teratospermia & 1.5 \\
\hline Asteno-teratospermia & 20.6 \\
\hline Oligo-asteno-teratospermia & 4.4 \\
\hline $\begin{array}{l}\text { Leukocytospermia (without other } \\
\text { pathologies defined by WHO) }\end{array}$ & 5.9 \\
\hline $\begin{array}{l}\text { Leukocytospermia (irrespective of all } \\
\text { other pathologies defined by WHO) }\end{array}$ & 19.1 \\
\hline
\end{tabular}

We rated sperm samples to be ROS-positive if they showed luminescence levels higher than 3000 RLUs per min (approximately $1.5 \times 10^{6}$ photons per min) calculated as average value from $15 \mathrm{~min}$ of measurement in the integrated mode. Based on the chemiluminescent measurement, we evaluated 33 sperm samples (48.5\%) as having elevated ROS concentrations (= ROS-positive samples). ROS-positive sperm samples were found in all subgroups of patients (data not shown). Tab. 2 summarises the proportion of ROS-positive sperm samples with respect to WHO-defined normo- and pathospermia.

Table 2. Distribution of ROS-positive sperm samples.

\begin{tabular}{|c|c|c|}
\hline Diagnosis & $\begin{array}{c}\text { ROS positive } \\
\text { samples } \\
(\%)\end{array}$ & $\begin{array}{c}\text { ROS negative } \\
\text { samples } \\
(\%)\end{array}$ \\
\hline $\begin{array}{c}\text { Normospermia } \\
\text { (15 patients })\end{array}$ & 20.0 & 80.0 \\
\hline $\begin{array}{c}\text { Pathospermia } \\
\text { (53 patients) }\end{array}$ & 56.6 & 43.4 \\
\hline
\end{tabular}

If we assess the numbers of leukocytes only (irrespective of all other criteria), WHO-defined leukocytospermia was found in 13 semen samples (19.1\%). Furthermore, 6 patients $(8.8 \%)$ had elevated levels of leukocytes in the semen but these numbers were below the WHO limit of leukocytospermia. Additionally, rare leukocytes were detected in 4 semen samples (5.9\%). Sperm samples contaminated by leukocytes were mostly ROS-positive, while a larger proportion of leukocyte-free sperm samples were interpreted to be ROS-negative (Fig. 1). However, large proportion of leukocyte-free sperm samples showed levels of ROS production comparable to sperm samples contaminated by leukocytes. Interestingly, some patients 


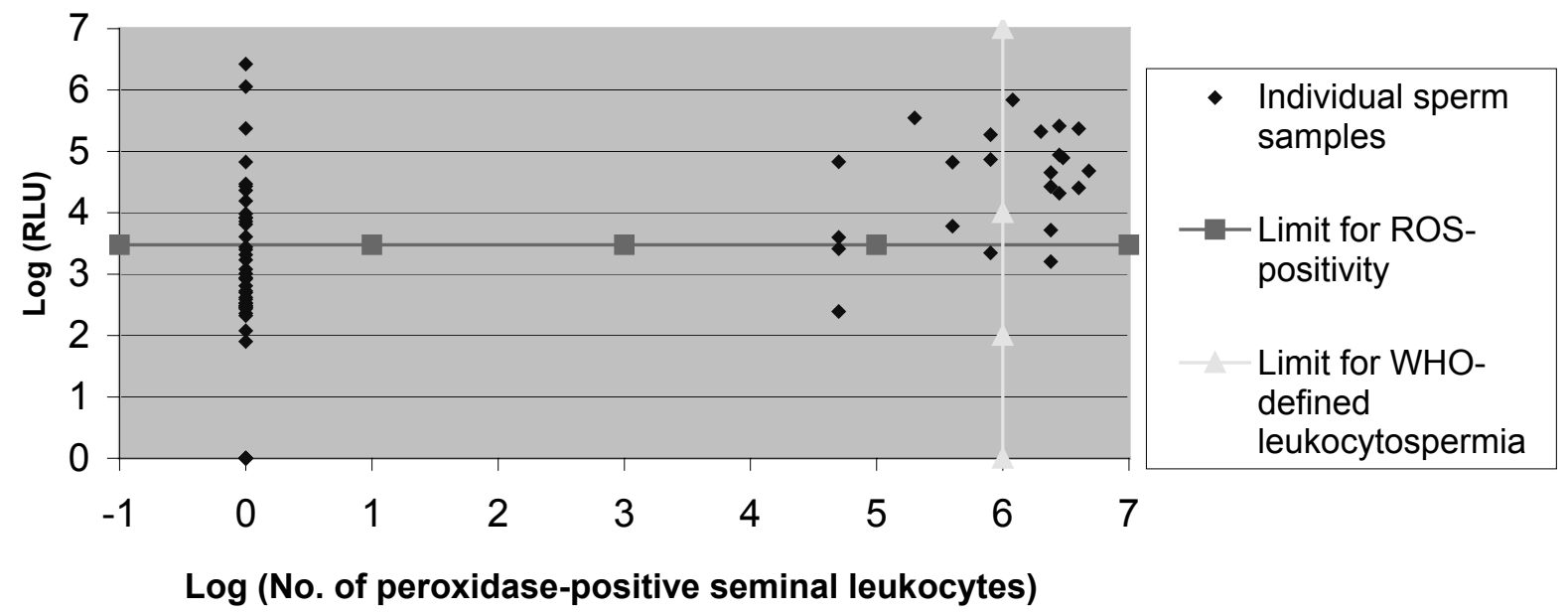

Fig. 1. ROS production in washed sperm suspensions with respect to seminal leukocyte contamination

with negative Endtz test had very high levels of produced ROS. No leukocytes were detected in 2 semen samples with the highest ROS levels.

Interrelationships between ROS production and other sperm pathologies were not assessed.

\section{DISCUSSION}

In spite of extensive research there is still lack of understanding of the factors contributing to normal or abnormal sperm function leading to infertility. The contemporary methods of semen analysis evaluates both production (e.g. concentration) and sperm quality (based on motility and morphology). However, the WHO approved schedule for routine sperm analysis provides little or no information about the genetic integrity of the spermatozoa. As a result, the cause of male infertility remains undiagnosed in up to half of all cases. Such a condition is called unexplained or idiopathic infertility ${ }^{11}$. One area of research that has been studied intensely during the past decade as a cause for male infertility is the role of OS. Spermatozoa were the first cell type suggested to generate highly reactive oxygen derived free radicals, ROS $^{2}$.

Excessive ROS production in the semen leads to deterioration of spermatozoa quality and function. However, our present state of knowledge does not allow us to define a cut-off limit between physiological and pathological levels of ROS. Elevated ROS level can indicate either semen contamination by leukocytes (especially granulocytes) or the occurrence of defective and/or immature spermatozoa. These possibilities can also be combined.

In the case of absence of seminal leukocytes it is obvious that spermatozoa produce excessive ROS. We found that spermatozoa in some patients produce very high quantities of ROS. In some cases, pure spermatozoa suspensions produced similar levels of ROS to those we could detect in washed sperm suspensions contaminated by leukocytes (see Fig. 1). Gil-Guzman et al. ${ }^{12}$ showed that levels of ROS production were negatively correlated with teratospermia and spermatozoa developmental stages. ROS production was found to be highest in the immature fraction of ejaculated sperms, which also contained sperm with abnormal head morphology and cytoplasmic retention. According to Baker et al. ${ }^{2}$, spermatozoa with cytoplasmic droplets and immature spermatozoa are mostly responsible for ROS production. For the future, it can be suggested that evaluation of morphological abnormalities should be done more carefully.

As for the second potential source of seminal ROS, neutrophils are normally present in human ejaculates at concentrations of less than 50000 cells $/ \mathrm{ml}$. In pathological situations (genital tract infection), the numbers of activated neutrophils are highly elevated and these white blood cells (WBC) seem to be responsible for impaired sperm physiology ${ }^{2}$. The diagnosis of leukocytospermia is usually based on the WHO definition of $1 \times 10^{6}$ WBC per $\mathrm{ml}$, but controversy also remains over the minimum leukocyte level that impairs fertility. Activated neutrophils may produce significant concentrations of ROS in amount less than $1 \times 10^{6} / \mathrm{ml}$ of seminal plasma. Oxidative stress can occur even in patients with very low seminal WBC counts (between 0 and $1 \times 10^{6} / \mathrm{ml}$ ). Therefore, it is not possible to determine a safe minimum WBC count. The presence of any WBC can be associated with oxidative stress and may therefore impair fertility ${ }^{13}$. Thus, it is questionable whether the limit for WHO-defined leukocytospermia is optimal for examination of men attending infertility centres. On the contrary Kaleli et al. ${ }^{14}$ suggest that leukocytospermia may have a favourable effect on some sperm functions at seminal leukocyte concentrations between 1 and $3 \times 10^{6} / \mathrm{ml}$.

In the case of rather low ROS production, it is difficult to assess whether the level of free oxygen radicals production is physiological or not. The appropriate production of ROS by spermatozoa is necessary for sperm capacitation, 
acrosome reaction and oocyte fusion ${ }^{15}$. Therefore, only excessive ROS production has a negative effect. Our limit value for ROS-positive sperm samples is still approximate. For this reason future studies on this important topic are urgently needed, including estimation of ROS production and total antioxidant capacity (TAC) of seminal plasma.

Apart from the ROS production, seminal plasma is rich in antioxidants that may protect spermatozoa from ROS-induced damage. The impaired antioxidant defence mechanisms in semen results in a decrease in TAC of the ejaculate ${ }^{16}$. Recent studies ${ }^{3,13,16,17}$ have resulted in the establishment of the ROS-TAC score as the optimal evaluation of OS level. ROS-TAC score is superior to ROS or TAC alone in discriminating between fertile and infertile men who have significantly lower ROS-TAC scores than controls ${ }^{17}$.

OS is defined as imbalance between ROS production and possible antioxidant protection (TAC). According to Aitken and Krausz ${ }^{18}$ OS is induced primarily by excessive ROS generation by immature and morphologically defective spermatozoa and/or by activated seminal leukocytes. Other possible OS sources include the redox cycling of xenobiotics, antioxidant depletion or apoptosis.

Recent advances in the field of male infertility have implicated oxidative stress and sperm DNA damage as one of major causative factors. Understanding of OS effects on human spermatozoa could improve the diagnosis of male infertility and could also specify the treatment strategy.

\section{ACKNOWLEDGEMENT}

This work was supported by grant IGA MZ $\check{C} R$ 2268-3 from the Ministry of Health of the Czech Republic.

\section{REFERENCES}

1. Aitken RJ. (1999) The Amoroso Lecture. The human spermatozoon: a cell in crisis? J Reprod Fertil 115, 1-7.

2. Baker MA, Krutskikh A, Aitken RJ. (2003) Biochemical entities involved in reactive oxygen species generation by human spermatozoa. Protoplasma 221, 145-151.

3. Sharma RK, Agarwal A. (1996) Role of reactive oxygen species in male infertility. Urology $48,835-850$.
4. Agarwal A, Saleh RA, Bedaiwy MA. (2003) Role of reactive oxygen species in the pathophysiology of human reproduction. Fertil Steril 79, 829-843.

5. Alvarez JG, Storey BT. (1995) Differential incorporation of fatty acids into and peroxidative loss of fatty acids from phospholipids of human spermatozoa. Mol Reprod Dev 42, 334-346.

6. Griveau JF, Le Lannou D. (1997) Reactive oxygen species and human spermatozoa: physiology and pathology. Int J Androl 20, 61-69.

7. de Laminarde E, Gagnon C. (1995) Impact of reactive oxygen species on spermatozoa: a balancing act between beneficial and detrimental effects. Hum Reprod 10, 15-21.

8. WHO laboratory manual for the examination of human semen and sperm-cervical mucus interaction, 4-th ed. New York: Cambridge University Press, 1999.

9. Saleh RA, Agarwal A, Kandirali E, Sharma RK, Thomas AJ Jr, Nada EA, Evenson DP, Alvarez JG. (2002) Leukocytospermia is associated with increased reactive oxygen species production by human spermatozoa. Fertil Steril 78, 1215-1224.

10. Shekarriz M, Sharma RK, Thomas AJ, Agarwal A. (1995) Positive myeloperoxidase staining (Endtz test) as a indicator of excessive reactive oxygen species formation in semen. J Assist Reprod Genet 12, 70-74.

11. Sherins RJ. How is male infertility defined? In: Robaire B, Pryor JL, Trasler JM, editors. Handback of Andrology. Lawrence: American Society of Andrology. Allen Press, 1995, ch. 15, p. 48-51.

12. Gil-Guzman E, Ollero M, Lopez MC, Sharma RK, Alvarez JG, Thomas AJ Jr, Agarwal A. (2001) Differential production of reactive oxygen species by subsets of human spermatozoa at different stages of maturation. Hum Reprod 16, 1922-1930.

13. Sharma RK, Pasqualotto AE, Nelson DR, Thomas AJ Jr, Agarwal A. (2001) Relationship between seminal white blood cell counts and oxidative stress in men treated at an infertility clinic. J Androl 22, 575-583.

14. Kaleli S, Ocer F, Irez T, Budak E, Aksu MF. (2000) Does leukocytospermia associate with poor semen parameters and sperm functions in male infertility? The role of different seminal leukocyte concentrations. Eur J Obstet Gynecol Reprod Biol 89, 185-191.

15. Zini A, de Lamirande E, Gagnon C. (1996) Low levels of nitric oxide promote human sperm capacitation in vitro. J Androl 16, 424-431.

16. Agarwal A, Saleh RA. (2002) Role of oxidants in male infertility: rationale, significance, and treatment. Urol Clin North Am 29, $817-27$.

17. Sharma RK, Pasqualotto FF, Nelson DR, Thomas AJ Jr, Agarwal A. (1999) The reactive oxygen species-total antioxidant capacity score is a new measure of oxidative stress to predict male infertility. Hum Reprod 14, 2801-2807.

18. Aitken RJ, Krausz C. (2001) DNA damage and Y chromosome. Reproduction 122, 497-506.

19. Carlsen E, Giwercman A, Keiding N, Skakkebaek NE. (1992) Evidence for decreasing quality of semen during past 50 years. BMJ 305, 609-613. 